\title{
A simple extended-cavity diode laser
}

\author{
A. S. Arnold, J. S. Wilson, ${ }^{\text {a) }}$ and M. G. Boshier \\ Sussex Centre for Optical and Atomic Physics, CPES, University of Sussex, Falmer, Brighton BN1 9QH, \\ United Kingdom
}

(Received 10 October 1997; accepted for publication 2 December 1997)

\begin{abstract}
Operating a laser diode in an extended cavity which provides frequency-selective feedback is a very effective method of reducing the laser's linewidth and improving its tunability. We have developed an extremely simple laser of this type, built from inexpensive commercial components with only a few minor modifications. A $780 \mathrm{~nm}$ laser built to this design has an output power of $80 \mathrm{~mW}$, a linewidth of $350 \mathrm{kHz}$, and it has been continuously locked to a Doppler-free rubidium transition for several days. (C) 1998 American Institute of Physics. [S0034-6748(98)01303-3]
\end{abstract}

\section{INTRODUCTION}

Diode lasers are now widely used in many experiments in optical and atomic physics. Although these devices are compact, simple, and relatively inexpensive, unmodified laser diodes do have some undesirable properties, mostly as a result of their short semiconductor cavity. In particular, their frequency is very sensitive to changes in temperature and injection current, and they have large linewidths $(\sim 100$ $\mathrm{MHz}$ ) and poor tunability. It is well known that these shortcomings can be rectified by operating the laser in a longer external cavity which provides frequency-selective optical feedback. ${ }^{1,2}$ A particularly simple implementation of this idea uses feedback from a diffraction grating mounted in the Littrow configuration. ${ }^{3,4}$ In this case the output facet of the diode must be antireflection coated to ensure stable operation in the presence of the strong feedback from the grating, but most laser diodes with output powers of more than $20 \mathrm{~mW}$ now have suitable coatings and a simple single-layer coating is easily applied to lasers with uncoated facets. ${ }^{5}$

In this article we describe a method for constructing an extended-cavity diode laser of this type. Its performance is similar to that of other designs, ${ }^{5-7}$ but it is particularly inexpensive and easy to build because it is based on simple modifications of a few commercial optical components.

\section{CONSTRUCTION}

The essential requirements for the extended cavity are that the laser diode, the diffraction grating, and a collimating lens all be located rigidly with respect to each other, and that the angle of the grating and the position of the lens be precisely adjustable. Our design (Fig. 1) takes advantage of the fact that these requirements can now be satisfied with convenient low-cost commercial components. ${ }^{8}$ A collimation tube (ThorLabs LT110P-B) holds the laser diode and the collimating lens (an aspheric optic with $f=6.2 \mathrm{~mm}$ and NA $=0.4$ ). The threaded tube also provides precisely adjustable focusing of the collimating lens, and it accurately locates the

\footnotetext{
a) Present address: Christ's College, Cambridge University, Cambridge CB2 3BU, United Kingdom.
}

collimating lens axis along the laser diode axis. The cavity itself is constructed on a mirror mount, eliminating the need for the milled baseplate with separate diode, collimating lens, and grating assemblies of other designs. The diffraction grating is mounted on the front plate of the mirror mount, and the collimation tube assembly on the back plate. The beam is coupled out of the cavity in the zeroth-order reflection from the grating. We have used the Newport Ultima U100-P mirror mount because of its good mechanical stability and because its thick plates are convenient for mounting the grating and collimation tube. The minor modifications made to the mirror mount are shown in Fig. 2. Two holes are tapped in the front plate of the mount and a square section of the plate is cut away. In addition, a clearance hole and clamping screw are added to the Ultima UPA-PA1 post adapter to mount the collimation tube. The total length of the extended cavity is about $20 \mathrm{~mm}$, which is long enough to reduce the linewidth below $1 \mathrm{MHz}$, while at the same time the corresponding mode spacing of $\sim 8 \mathrm{GHz}$ is large enough to give a useful continuous scan range and robust singlefrequency operation.

The design shown in Figs. 1 and 2 has a third component, a grating mount (Fig. 3) which is screwed to the front plate of the mirror mount. It is possible to dispense with this extra component and simply mill the plate of the mirror mount directly to the Littrow angle. This further simplifies the design, although the focusing adjustment can become awkward for such a short cavity.

Our standard diffraction grating for near-infrared operation is a gold-coated 1800 lines/mm holographic grating on a $15 \times 15 \times 3 \mathrm{~mm}^{3}$ substrate (Richardson Grating Laboratory 35-83-X-330). With the light polarized parallel to the lines of the grating it provides $20 \%$ feedback, sufficient for good tunability and stability with many laser diodes, while the $80 \%$ output coupling gives high output power. The grating is mounted on a $16 \mathrm{~mm}$ diameter $\times 2 \mathrm{~mm}$ thick disk piezoelectric transducer (PZT) to provide fine adjustment of the cavity length for scanning the laser frequency.

An advantage of the compact cavity design is that it is easy to control the temperature of the complete laser using a Peltier thermoelectric cooler (TEC). To this end, a small hole 


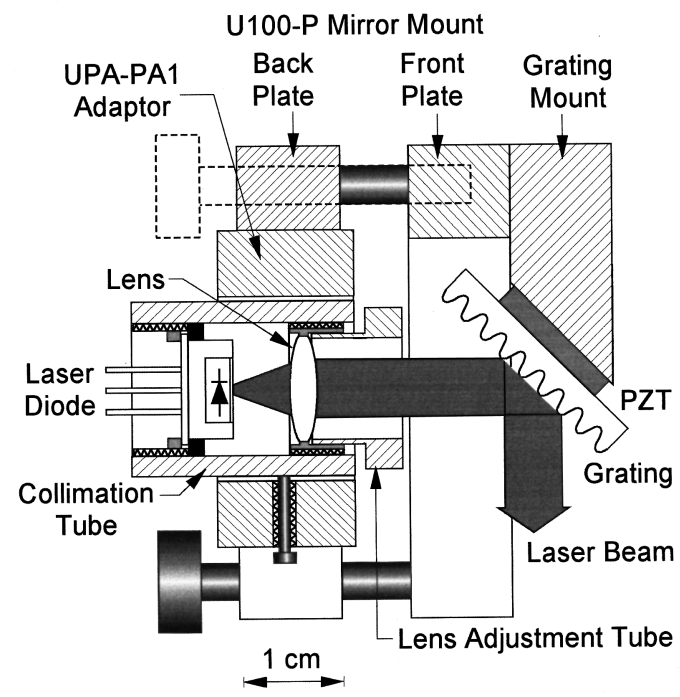

FIG. 1. Schematic diagram of the extended-cavity laser, viewed from above. The Peltier thermoelectric cooler and mounting base are not shown.

is drilled in the mirror mount near the diode, and a thermistor or other temperature sensor glued inside it. A thin metal plate is glued between the base of the mirror mount and the TEC to provide a good thermal connection between the laser and the TEC. We use a $30 \times 30 \mathrm{~mm}^{2}$ TEC with $33 \mathrm{~W}$ of cooling power, in which case the dimensions of the plate are $3 \times 30$ $\times 40 \mathrm{~mm}^{3}$ (a clearance hole must also be drilled in the plate to accommodate a raised bolt head on the mirror mount). The other side of the TEC must be attached to a suitable heatsink. If the laser is to be mounted on an optical table, a standard slotted base (e.g., ThorLabs BA2) works well. All glued joints should be made with a low vapor pressure epoxy such as Torr-Seal to avoid outgassing contamination if the laser is to be operated in a sealed enclosure.

Alignment of the completed laser proceeds as follows. The laser diode is mounted in the collimation tube, and the lens is adjusted to collimate the beam over a distance of several meters. The collimation tube is then clamped in the

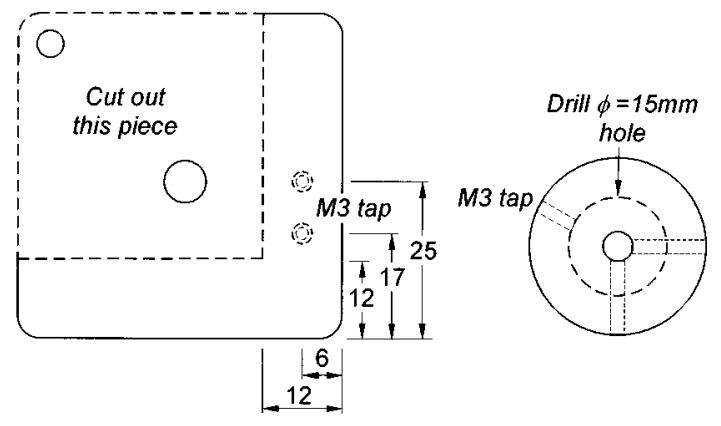

U100-P Front Plate

UPA-PA1 Adapter

FIG. 2. The modifications made to the u100-P mirror mount. On the front plate of the mirror mount (left), the section bordered with dashed lines is cut out and two tapped holes are added to attach the grating mount. The center hole of the PA1 adapter (right) is drilled out to $15 \mathrm{~mm}$ and a tapped hole is added to take the screw which clamps the collimation tube. All dimensions are in $\mathrm{mm}$.
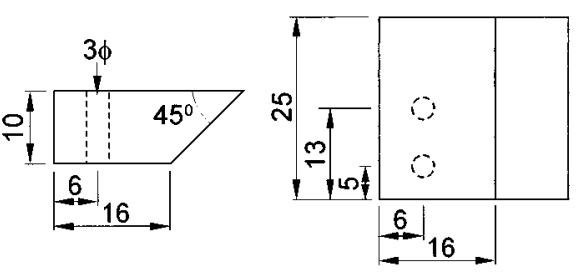

FIG. 3. The aluminium grating mount used with an 1800 lines/mm grating at a wavelength of $780 \mathrm{~nm}$. Top view (left) and front view (right). All dimensions are in $\mathrm{mm}$.

mirror mount as shown in Fig. 1, rotated so that the long axis of the elliptical laser beam is perpendicular to the lines on the grating. Next, the laser current is reduced just below threshold for the solitary laser diode, where the diode is most sensitive to feedback as it cannot lase by itself. Adjusting the mirror mount to direct the Littrow reflection from the grating back into the center of the collimating lens should cause a second beam to become visible near the main zeroth-order output beam. This beam is much weaker than the main one because it has made a complete round trip inside the cavity. Collapsing it into the main output beam by further grating adjustments should then produce a large increase in output power as the feedback from the grating takes the laser above threshold. The output power can be optimized by adjusting the grating angle and the focusing of the collimation lens. The lens adjustment is very critical, corresponding to only a few degrees of rotation of the threaded lens mount. While it can be accomplished by rotating the lens carefully with a small screwdriver, we have found it more convenient to glue to the lens mount a short adjustment tube, extending beyond the end of the collimation tube (Fig. 1). Once the system is lasing, the laser current can be increased to give the desired output power, and the horizontal grating adjustment is used to tune the laser to the required wavelength.

\section{PERFORMANCE}

We have constructed several systems using Spectra Diode Labs SDL-5401 $780 \mathrm{~nm} 50 \mathrm{~mW}$ laser diodes. These devices perform well in the cavity described here and were used to obtain most of the performance data presented in this section.

The short-term linewidth of the free-running laser was measured by two methods. First, two similar lasers were heterodyned, with the width of the beatnote yielding a combined linewidth of less than $1 \mathrm{MHz}$ for a $200 \mathrm{~ms}$ averaging time. Second, the linewidth was measured by monitoring the fluctuations in the light transmitted by a Fabry-Perot etalon with the laser tuned approximately half way up a transmission peak. This gave a linewidth of $350 \mathrm{kHz}$ for a $200 \mathrm{~ms}$ averaging time. The associated power spectral density is shown in Fig. 4. This performance is typical of short extended-cavity diode lasers and demonstrates the excellent mechanical stability of the mirror mount used in our design as a cavity. Linewidths below a few $\mathrm{MHz}$ are only obtained when the laser is driven by a current supply having very low 


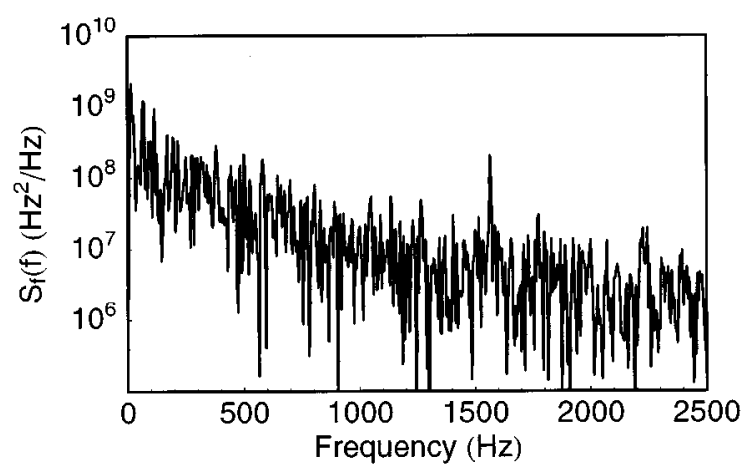

FIG. 4. The power spectral density $S_{f}(f)$ of the free-running laser's frequency fluctuations. The corresponding laser linewidth is $350 \mathrm{kHz}$.

noise, typically less than a few $\mu \mathrm{A}$. This figure may not be met by many low-cost commercial diode laser power supplies without substantial additional low-pass filtering. As an alternative, a simple power supply comprising a $12 \mathrm{~V}$ leadacid car battery, a series potentiometer to control the current, and a few protection components provides excellent performance. If line-powered power supplies are used, care must also be taken to avoid laser damage from transients caused by switching fluorescent lights. In particular, the lowestimpedance path between any grounded points in the circuit and the power supply should not pass through the laser diode.

The laser also has good long-term stability when it is temperature stabilized (to $10 \mathrm{mK}$ ) and placed in a box which excludes draughts. A laser stabilized passively in this way and then locked to the peak of a rubidium saturatedabsorption line with a simple servo loop remained locked continuously for several days. The lock was eventually lost through laser frequency shifts associated with atmospheric pressure changes. Enclosing the locked laser in a hermetically sealed box reduced the drifts which must be taken out by the servo loop to less than $500 \mathrm{MHz}$ over a few weeks, further improving the long-term reliability of the lock.

Continuous scans of $8 \mathrm{GHz}$ (the cavity mode spacing) can be achieved using the PZT alone, with even larger continuous scans expected with appropriate synchronous translation and rotation of the grating. ${ }^{9}$ The laser can also be tuned discontinuously over a range of about $20 \mathrm{~nm}$ around the free-running wavelength of the solitary laser diode by rotating the grating. This usually causes the laser to mode hop a few times in steps of $5-8 \mathrm{GHz}$ (roughly equal to the extended-cavity mode spacing), before making a much larger mode hop corresponding to the $50 \mathrm{GHz}$ free spectral range of the solitary laser diode cavity. The resulting "holes" in the tuning curve can be shifted away from a region of interest by adjusting the laser temperature. The frequency of the solitary SDL-5401 laser diode tunes with temperature at about 35 $\mathrm{GHz} / \mathrm{K}$.

Much smoother tuning is expected from a laser which has a lower reflectivity output facet. In the case of laser diodes supplied with uncoated facets, a very low reflectivity can be obtained from a single-layer coating applied using a simple technique. ${ }^{5}$ We have coated several Hitachi HL7806G $780 \mathrm{~nm} 5 \mathrm{~mW}$ diodes using this method, and found that these diodes do indeed have very much smaller holes in their tuning curves. As a demonstration of this improved tuning we have assembled several laser systems with these lasers, and tuned all of them to the rubidium D2 line within a few minutes by looking for fluorescence from a vapour cell without any specific adjustment of the laser current or temperature. These systems do have lower output power than those using the SDL-5401 lasers, but their smooth tunability can be readily transferred to much higher power devices by injection locking.

Finally, we discuss output power. It is well known that laser diodes fail suddenly and irreversibly as their output power is increased. Until recently the true maximum safe output power was usually quite close to the limit given by the manufacturer. However, this situation has changed, and it now appears that many commercial laser diodes can be run at output powers which considerably exceed the manufacturer's specifications. This true maximum power can be found by imposing a large amplitude $10 \mathrm{kHz}$ ac current modulation on the drive to the laser, and then watching for the appearance of a roll-off in the light versus current characteristic of the solitary laser diode as the maximum current is slowly increased above the manufacturer's specified maximum. ${ }^{10}$ The fast modulation and large amplitude of the ac current ensures that the time spent at destructive powers is short enough to avoid permanent damage. The onset of the roll-off indicates the maximum current at which the diode can be operated without damage. We use a modulation amplitude of at least $30 \mathrm{~mA}$ for testing devices with a threshold of $30 \mathrm{~mA}$ and nominal maximum current of $70 \mathrm{~mA}$. Several of the nominally $50 \mathrm{~mW}$ SDL-5401 laser diodes have been tested in this way, and found to exhibit a wide range of true maximum powers, from $55 \mathrm{~mW}$ up to $200 \mathrm{~mW}$. One of these laser diodes has been operated in the extended-cavity configuration described here, delivering an output power of $80 \mathrm{~mW}$ off the grating. We have also found that most of the nominally $5 \mathrm{~mW}$ Hitachi HL7806G lasers can be operated at 15 $\mathrm{mW}$, giving an output power of over $10 \mathrm{~mW}$ from the extended cavity. This is sufficient for many experiments in laser cooling and trapping.

\section{ACKNOWLEDGMENTS}

This work was supported by the UK Engineering and Physical Sciences Research Council and the University of Sussex. One of the authors (A.A.) is grateful to the Association of Commonwealth Universities for a Commonwealth scholarship.

${ }^{1}$ C. E. Wieman and L. Hollberg, Rev. Sci. Instrum. 62, 1 (1991).

${ }^{2}$ P. Zoorabedian, in Tunable Lasers Handbook, edited by F. J. Duarte (Academic, London, 1995).

${ }^{3}$ M. W. Flemming and A. Mooradian, IEEE J. Quantum Electron. 17, 44 (1981).

${ }^{4}$ R. Wyatt and W. J. Devlin, Electron. Lett. 19, 110 (1983).

${ }^{5}$ M. G. Boshier, D. Berkeland, E. A. Hinds, and V. Sandoghdar, Opt. Commun. 85, 355 (1991). 
${ }^{6}$ K. B. MacAdam, A. Steinbach, and C. Wieman, Am. J. Phys. 60, 1098 (1992).

${ }^{7}$ L. Ricci, M. Weidemüller, T. Esslinger, A. Hemmerich, C. Zimmermann, V. Vuletic, W. König, and T. W. Hänsch, Opt. Commun. 117, 541 (1995).

${ }^{8}$ Throughout this article we provide details of the commercial components which we have used, in order to assist the reader who wishes to duplicate our system. Components from other manufacturers may of course deliver similar or better performance.

${ }^{9}$ P. McNicholl and H. J. Metcalf, Appl. Opt. 24, 2757 (1985).

${ }^{10}$ K. Gibble, Proceedings of the 1997 NASA/JPL Fundamental Physics in Microgravity Workshop, Santa Barbara, 7-9 May 1997. 